\title{
Die Bedeutung der Gestaltung des Lernfelds und des Funktionsfelds für den subjektiven Erfolg betrieblicher Weiterbildung
}

\author{
Anita Sandmeier (D) • Ulrike Hanke • Martin Gubler
}

Eingegangen: 30. Januar 2018 / Angenommen: 28. April 2018 / Online publiziert: 18. Mai 2018

(C) Der/die Autor(en) 2018

Zusammenfassung Die Frage, wie der kurz- und der langfristige Erfolg von betrieblichen Weiterbildungen positiv beeinflusst werden kann, ist für Unternehmen von großer Bedeutung. Aktuell werden in der Praxis jedoch kaum Evaluationen getätigt, die über eine summative Evaluation hinausgehen. Der vorliegende Beitrag untersucht ausgehend von einem praxistauglichen Rahmenmodell den Zusammenhang verschiedener Merkmale des Lernfelds (d.h. der Weiterbildung) und des beruflichen Funktionsfelds auf den subjektiv eingeschätzten Erfolg in verschiedenen betrieblichen Weiterbildungen $(N=477)$. Die Regressionsanalysen zeigen, dass der kurzfristige Erfolg insbesondere mit der Gestaltung der Weiterbildung zusammenhängt. Der langfristige Erfolg hingegen ist hingegen eher abhängig von der Gestaltung des beruflichen Umfelds.

Schlüsselwörter Betriebliche Weiterbildung · Gestaltung von Weiterbildung · Lerntransfer · Arbeitskontext · Evaluation

Publisher's Note Springer Nature remains neutral with regard to jurisdictional claims in published maps and institutional affiliations.

Prof. Dr. A. Sandmeier $(\bowtie) \cdot$ Prof. Dr. M. Gubler

Institut für Professionsforschung und Personalentwicklung, Pädagogische Hochschule Schwyz, Zaystrasse 42, 6410 Goldau, Schweiz

E-Mail: anita.sandmeier@phsz.ch

Prof. Dr. M. Gubler

E-Mail: martin.gubler@phsz.ch

PD Dr. U. Hanke

Teachertraining, Im Käppelefeld 39, 79189 Bad Krozingen, Deutschland

E-Mail: mail@ulrike-hanke.de 


\title{
The relevance of the «learning field» and «performance field» on the subjectively perceived success of continuing vocational education and training
}

\begin{abstract}
The question how to improve the short-term as well as the long-term success of continuing vocational education and training (CVET) is of great importance for companies. Currently, however, evaluations in practice are still often only of a summative nature. Based on a practically applicable model and empirical data from participants in a wide range of CVET courses $(N=477)$, this paper presents various factors organisations can use to affect the subjective success of CVET, both in the "learning field" (i.e., the CVET activity itself) and the "performance field" (i.e., the work context). Regression analyses indicate the short-term success is mainly related to the quality of the CVET activity, whereas long-term success is more dependent on the quality of the performance field.
\end{abstract}

Keywords Continuing vocational education and training $\cdot$ Design of continuing vocational education and training $\cdot$ Learning transfer . Work context - Evaluation

\section{Einleitung}

Betriebliche Weiterbildungen haben das Potenzial, das Wissen, die Kompetenzen und Fähigkeiten von Mitarbeitenden zu verbessern und dadurch den organisationalen Erfolg zu erhöhen (Blume et al. 2010). Unternehmen investieren Jahr für Jahr sehr viel Geld in Weiterbildung, in der Schweiz jährlich geschätzte 1,5 Mrd. CHF. Dies ist ein europäischer Spitzenwert, der nur in Österreich und Schweden übertroffen wird (Bundesamt für Statistik 2014). Um den Erfolg von Weiterbildung zu messen, stehen den Firmen verschiedene Maßnahmen zur Verfügung. In der Praxis versuchen viele Unternehmen, die Qualität von Weiterbildungen mit Feedback (happy sheets) oder einem Wissenstest direkt nach dem Training zu sichern. Dabei wird jedoch nur die Weiterbildung beurteilt; eine Einschätzung der Passung der Weiterbildung zur beruflichen Praxis und den dort gegebenen Rahmenbedingungen wird seltener evaluiert. Lediglich $10 \%$ der Firmen in Deutschland geben an, dass sie Transfersicherung betreiben und die Gegebenheiten im Anwendungsfeld mit in die Evaluation einbeziehen (Käpplinger 2009).

Der theoretische Diskurs der Transfer- und Lernforschung konzentriert sich aktuell stark auf die Frage der Erfassung transferrelevanter Faktoren. Ausgehend vom durch Holton III et al. (2000) entwickelten Inventar „LTSI Learning Transfer System Inventory“ entstanden zahlreiche Studien, die insbesondere die psychometrischen Eigenschaften des Inventars in verschiedenen Sprachen untersuchen (z. B. Devos et al. 2007; Kauffeld et al. 2008). Ein weiterer Schwerpunkt des wissenschaftlichen Diskurses liegt bei der Erforschung der Transfermotivation des Individuums (z. B. Massenberg et al. 2017) und bei der Gestaltung eines günstigen Transferumfelds (z.B. Chatterjee et al. 2018). 
Die Diskurse in der Praxis und der Wissenschaft finden etwas abgekoppelt voneinander statt. Die wissenschaftlichen Erkenntnisse sind zu unübersichtlich und zu unklar, um praktische Relevanz zu entfalten (Grossman und Salas 2011). Gessler (2012) machte mit seinem Rahmenmodell zum Lerntransfer beruflicher Weiterbildungen einen Vorschlag, der auf wissenschaftlichen Erkenntnissen aufbaut, jedoch auch die Bedürfnisse der Praxis (ökonomische Erhebung von transferrelevanten Faktoren) aufnimmt. Im Modell liegt der Fokus auf jenen Faktoren, die von den Organisationen kontrolliert werden können, d.h. Faktoren der Gestaltung der Weiterbildung (Lernfeld) und des Arbeitsumfelds, in dem das Gelernte angewendet werden soll (Funktionsfeld).

Der vorliegende Beitrag untersucht, welchen Einfluss die Faktoren in diesen beiden Feldern auf den subjektiv eingeschätzten kurz- und langfristigen Erfolg der Weiterbildung haben. Ausgehend von der Frage, wie der Erfolg einer Weiterbildungsmaßnahme gemessen werden kann, werden das Rahmenmodell von Gessler (2012) vorgestellt und die relevanten Faktoren im Lern- und Funktionsfeld näher beschrieben. Im empirischen Teil wird der differenzielle Einfluss dieser Felder auf die affektive Zufriedenheit, die Nützlichkeitseinschätzung, das Gefühl, etwas gelernt zu haben, die Anwendung im konkreten beruflichen Kontext und die subjektiv eingeschätzten Auswirkungen auf die Organisation untersucht.

\section{Theoretischer Rahmen und Forschungslage}

Der Erfolg von betrieblicher Weiterbildung kann summativ oder formativ evaluiert werden. Eine summative, ergebnisorientierte Evaluation untersucht die Qualität oder den Nutzen einer Weiterbildung; eine formative Evaluation will prozessorientiert Stärken und Schwächen entdecken mit dem Ziel, die Weiterbildung zu verbessern (Scriven 1991).

\subsection{Ergebnis- und prozessorientierte Modelle}

Ergebnisorientierte Modelle fokussieren primär auf den Output einer Weiterbildungsmaßnahme. Der Output wird dabei oft durch die vier Stufen des in der betrieblichen Praxis am weitesten verbreiteten Instruments zur Weiterbildungsevaluation von Kirkpatrick (1979) bestimmt. Aus Zeit- und Kostengründen messen Unternehmen meist nur Reaktion und den Lernerfolg direkt nach der Weiterbildung und vernachlässigen die übrigen Stufen (Grohmann und Kauffeld 2013). Zusätzlich fehlt den Betrieben oft auch das Know-how, für jede Weiterbildung einen Fragebogen zu entwickeln, der die Anwendung in der Praxis und die Resultate der Weiterbildungen psychometrisch einwandfrei misst. Grohmann und Kauffeld (2013) entwickelten ein generisches Instrument (Q4TE), das für eine Vielfalt von verschiedenen Weiterbildungen eingesetzt werden kann und alle vier Stufen Kirkpatricks abdeckt. In diesem Instrument schlagen sie ausgehend von Wang und Wilcox (2006) vor, kurzfristigen und langfristigen Erfolg von Weiterbildungen zu unterscheiden. Im kurzfristigen Erfolg sind die Reaktion und Einschätzungen der Teilnehmenden direkt nach der Weiterbildung zusammengefasst, d. h. die affektive Zufriedenheit, die 
Nützlichkeitseinschätzung und das Gefühl, etwas gelernt zu haben. Im langfristigen Erfolg werden die Anwendung im konkreten beruflichen Kontext und die subjektiv eingeschätzten Auswirkungen auf die Organisation gemessen (Grohmann und Kauffeld 2013). Das Instrument ist geeignet, den subjektiven Erfolg einer Weiterbildung differenziert zu erfassen. Es kann aber keine Aussage dazu machen, wie die Ergebnisse entstehen und über welche Faktoren sie systematisch optimiert werden können (Gessler 2012).

\subsection{Was beeinflusst den Lerntransfer?}

Ausgehend von Überlegungen von Baldwin und Ford (1988) entwickelte Holton III (1996, 2005) ein umfassendes Modell von 16 transferrelevanten Einflussfaktoren und ein Frageninventar, um diese zu messen (LTSI Learning Transfer System Inventory - Holton III et al. 2000). Das Modell geht davon aus, dass der Lerntransfer abhängt von der Fähigkeit und der Motivation der Weiterbildungsteilnehmenden und von Umweltfaktoren. Es gibt bislang jedoch nur wenige Studien, die die prädiktive Validität, d.h. den Einfluss dieser Faktoren auf den tatsächlichen Transfer untersuchen (Hutchins et al. 2013). Hutchins et al. (2013) erforschten den Zusammenhang der LTSI-Faktoren auf die Transferintention und stellten dabei fest, dass ausser der Transfermotivation keiner der Faktoren einen statistisch signifikanten Beitrag leistete.

Aus Sicht von Grossman und Salas (2011) ist die Anzahl von möglichen Einflussfaktoren unübersichtlich, die Datenlage ambivalent und dadurch für die Praxis verwirrend. Sie plädierten vor diesem Hintergrund dafür, die Analysen zu vertiefen, indem nicht mehr alle möglichen Faktoren in ihrem Einfluss auf den Lerntransfer untersucht werden, sondern nur noch jene, die sich in Metaanalysen als die wichtigsten herausgestellt hatten. Sie entwickelten ein Modell, das die wesentlichen Faktoren zusammenfasst und damit Unternehmen ein Instrument an die Hand gibt, um bei der Konzeption von betrieblichen Weiterbildungen die wichtigsten Stellschrauben hinsichtlich des Lerntransfers zu berücksichtigen. Sie kategorisierten die Einflussfaktoren in die drei Kategorien Teilnehmercharakteristika, Trainingsdesign und Arbeitsumgebung. Auf dieser Basis entwickelte Gessler (2012) ein Rahmenmodell zur Ermittlung des Lerntransfers. Sein Fokus lag dabei auf jenen Faktoren, die von den Organisationen kontrolliert werden können, nämlich Faktoren der Weiterbildung (Lernfeld) und Faktoren des Arbeitsumfelds, in dem das Gelernte angewendet werden soll (Funktionsfeld).

Der vorliegende Beitrag folgt diesem Ansatz und beschränkt sich in der Folge auf die von Gessler (2012) und Hinrichs (2016) begründete Auswahl an Faktoren. Im Lernfeld die (1) methodische Gestaltung der Weiterbildungsmaßnahme, (2) die Kompetenzunterstützung, (3) die Situiertheit, (4) die Transferorientierung und (5) die Transfermotivation der Teilnehmenden. Im Funktionsfeld die Faktoren (1) Transferunterstützung durch die vorgesetzte Person, (2) Transferklima und (3) Transferkapazität der Situation.

Ausgehend von der von Grohmann und Kauffeld (2013) gemachten Differenzierung geht diese Studie den Fragen nach, (1) welche Faktoren mit dem kurzfristigen und welche mit dem langfristigen Erfolg einer Weiterbildung zusammenhängen und 


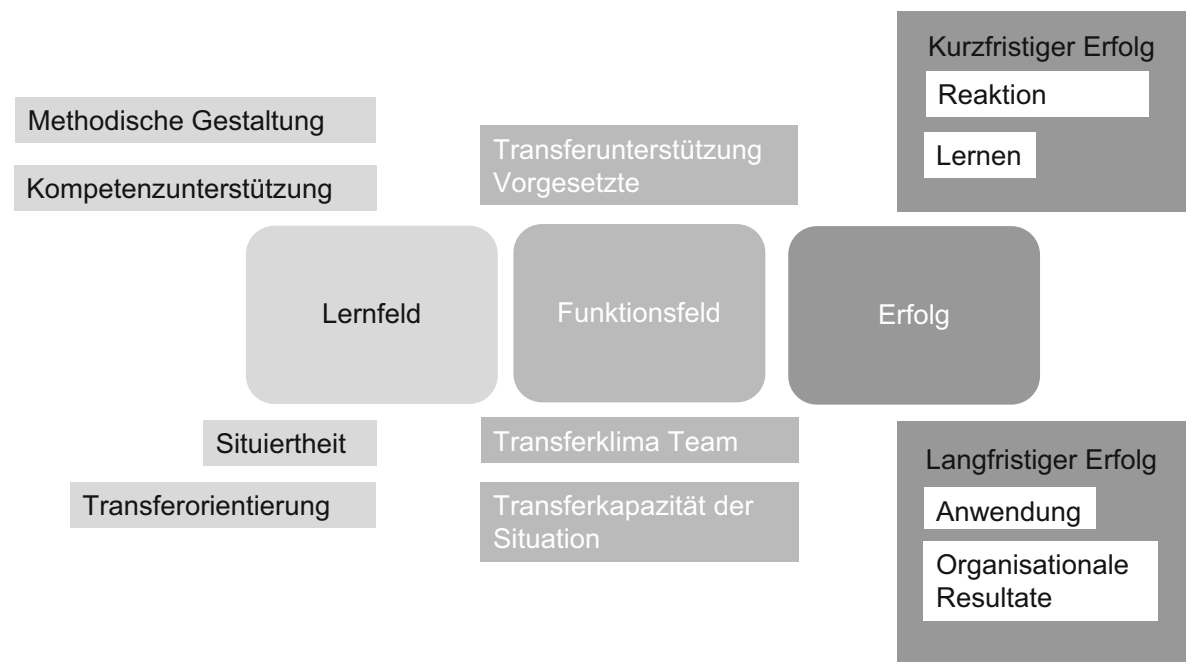

Abb. 1 Faktoren des Lernfelds und des Funktionsfelds mit erwartetem Zusammenhang mit dem kurzund langfristigen Erfolg von Weiterbildungen (in Anlehnung an Gessler 2012)

(2) ob es sich dabei um die gleichen oder um unterschiedliche Faktoren handelt. In den nachfolgenden Analysen wurde die Transfermotivation der Teilnehmenden nicht als Einflussfaktor einbezogen, da der Fokus auf den Umweltfaktoren liegt, die durch das Unternehmen gestaltbar sind (s. Abb. 1).

\subsubsection{Faktoren des Lernfelds}

Ausgehend von zahlreichen Befunden aus der Lern-, Motivations- und Unterrichtsforschung wird davon ausgegangen, dass die methodische Gestaltung von Weiterbildungsmaßnahmen Einfluss hat auf die Zufriedenheit der Teilnehmenden mit der Maßnahme und auf ihren Wissenserwerb (Wang und Wilcox 2006). In diesem Kontext ist es vor allem bedeutsam, dass die Lernumgebung und ihre Gestaltung sich wesentlich an den jeweiligen Lernenden orientiert (Gagné et al. 2005; Hanke 2005). Der Grad der Abwechslung, Möglichkeiten des Austauschs unter den Teilnehmenden, der sinnvolle Einsatz von Medien und die verständliche Anleitung durch den Trainer sind dabei wesentliche Faktoren.

Ebenso wichtig ist es, dass das Lernfeld Kompetenzunterstützung bietet, indem es Kompetenzerfahrungen ermöglicht. Dafür ist es wichtig, dass Leistungen anerkannt und beachtet, Gelegenheiten zum Üben geboten und Feedback zum Fortschritt gegeben werden (Prenzel und Drechsel 1996). Das in der Literatur am häufigsten aufgeführte Unterstützungsinstrument für den Transfererfolg ist das Feedback. Die Metastudie von Hattie (2013) hebt die Rolle des Feedbacks für den Lernerfolg und einen gelingenden Transfer hervor. Die Motivationsforschung zeigt zudem, dass Menschen am besten lernen, wenn dies in einer grundlegend wertschätzenden Umgebung stattfindet (Deci und Ryan 1993). 
Unter Situiertheit der Weiterbildung wird verstanden, dass in der Weiterbildung an die Erfahrungen der Teilnehmenden angeknüpft und diese explizit einbezogen werden. Dies gilt als eine wesentliche Voraussetzung für das erfolgreiche Lernen von Erwachsenen. Ihr Lernen baut stets auf bekanntem Wissen und bereits erworbenen Handlungspotenzialen auf und ist ohne diesen Bezug nicht erfolgreich (Lipowsky 2004).

Als viertes erweist sich die Transferorientierung der Weiterbildung zentral für den Lerntransfer. Um Transferorientierung sicherzustellen, ist schon in der Weiterbildungsmaßnahme selbst die Anwendungssituation zu thematisieren, z. B. durch praxisnahe und handlungsorientierte Aufgabenstellungen. Bhatti et al. (2014) zeigten z. B. konkret auf, dass die Weiterbildungsinhalte einen direkten Effekt auf die affektive Reaktion der Teilnehmenden haben. Auch Lieberman und Hoffmann (2008) konnten die wahrgenommene praktische Relevanz des Trainings als wesentlichen Einflussfaktor für die Zufriedenheit der Teilnehmenden identifizieren.

Aus den dargelegten Überlegungen und Studien werden folgende beiden Hypothesen abgeleitet:

H1 Die Faktoren des Lernfelds stehen insbesondere mit dem kurzfristigen Erfolg der Weiterbildung (Reaktion und Lernen) im Zusammenhang.

Da die Transferorientierung die Voraussetzung dafür ist, dass die Teilnehmenden Transfersituationen in der Praxis erkennen, sollte sie zusätzlich einen Einfluss auf den langfristigen Erfolg der Weiterbildung haben.

H2 Die Transferorientierung einer Weiterbildung hat zusätzlich auch einen Einfluss auf den langfristigen Erfolg einer Weiterbildung.

\subsubsection{Faktoren des Funktionsfelds}

Im Funktionsfeld, also der beruflichen Anwendungsumgebung, zeigen sich insbesondere der Vorgesetzte, das Klima im Team und in der Organisation sowie die Kapazität der Situation als bedeutsame Faktoren für den langfristigen Erfolg einer Weiterbildung (Grossman und Salas 2011).

Damit neu Gelerntes in den Arbeitsalltag integriert werden kann, muss in Unternehmen eine Kultur vorherrschen, die dies ermöglicht. Zentral sind dabei die Vorgesetzten. Stehen diese Neuem kritisch gegenüber, werden die Mitarbeitenden nur zögerlich oder gar nicht wagen, Neues einzubringen. So konnte z. B. Foxon (1997) zeigen, dass die wahrgenommene Unterstützung durch den Vorgesetzten mit einer höheren Transferleistung der ehemaligen Weiterbildungsteilnehmenden korrelierte (s. auch Martin 2010). Blume et al. (2010) bestätigten dies in ihrer Metaanalyse. Dabei scheint es so zu sein, dass der Vorgesetzte vor allem die Transfermotivation der Mitarbeitenden beeinflusst, was wiederum eine Voraussetzung für erfolgreichen Transfer ist (Bhatti et al. 2014). Weitere Evidenz für den Zusammenhang zwischen der Unterstützung durch Vorgesetzte und die Transferleistung der Mitarbeitenden liefern die Studien von Festner (2012), Lee et al. (2014) sowie Ng und Ahm (2011). 
Auch das Transferklima im Team beeinflusst, wie frei sich Mitarbeitende fühlen, Neues einzubringen. Colquitt et al. (2000) zeigen auf, dass zwischen dem Transferklima und dem Lerntransfer ein wesentlicher Zusammenhang besteht (s. auch Burke und Hutchins 2008; Blume et al. 2010).

Ein weiterer Einflussfaktor auf den Lerntransfer ist die Transferkapazität der Situation. Finden Mitarbeitende kein Setting vor, in dem sich das neu Gelernte nutzen lässt, können sie es auch nicht anwenden. So konnte Clarke (2002) fehlende bzw. nur unzureichend vorhandene Ressourcen und Möglichkeiten, das Gelernte im Transferfeld anzuwenden, als die wichtigste Transferbarriere identifizieren. Diesen Befund unterstützt die Studie von Lim und Johnson (2002). In dieser Studie bewerteten die Teilnehmenden ausreichend vorhandene Anwendungs- und Umsetzungsmöglichkeiten als das zentrale Unterstützungsinstrument für gelingenden Transfer.

Zusammenfassend stehen die Faktoren des Funktionsfelds - die Unterstützung durch Vorgesetzte, Transferklima im Team und die Transferkapazität - vor allem mit dem langfristigen Erfolg in Zusammenhang. Für den kurzfristigen Erfolg, d.h. die Reaktion und das Lernen, sollten diese Faktoren weniger Relevanz haben.

H3 Die Faktoren des Funktionsfelds stehen in keinem Zusammenhang mit dem kurzfristigen Erfolg einer Weiterbildung.

H4 Die Faktoren des Funktionsfelds haben Einfluss auf die Faktoren des langfristigen Erfolgs einer Weiterbildung.

\section{Untersuchungsdesign}

Das Projekt „Der Wert der Weiterbildung“, das von der Schweizer Kommission für Technologie und Innovation (KTI) finanziert wurde, hatte das Ziel, ein praxistaugliches, wissenschaftsbasiertes Instrument zur formativen Evaluation betrieblicher Weiterbildungen zu entwickeln. Dafür wurden schrittweise in drei aufeinanderfolgenden Phasen bestehende, wissenschaftlich validierte Instrumente (Grohmann und Kauffeld 2013; Hinrichs 2014; Kauffeld et al. 2008) kombiniert und gekürzt, basierend auf psychometrischen Eigenschaften und der Einschätzung der praktischen Relevanz der Items von Berufsleuten aus der HR-Praxis.

\subsection{Studien- und Stichprobenbeschreibung}

Die Daten der vorliegenden Untersuchung stammen aus der zweiten Phase des Projekts. Sie wurden im Juli 2016 erhoben, indem retrospektiv die betrieblichen Weiterbildungen von drei grossen Schweizer Unternehmen evaluiert wurden, die nicht länger als ein Jahr zurücklagen. Die Trainings waren thematisch sehr unterschiedlich, von technischen Schulungen bis zur Führungsentwicklung, und dauerten alle mindestens zwei Tage.

Bei der Befragung handelte es sich um eine Querschnittsbefragung, die standardisiert und elektronisch stattfand. Insgesamt wurden 1436 Links verschickt; 590 Personen beendeten die Umfrage, was einem Rücklauf von 41,1\% entspricht. Die 
hier präsentierten Analysen basieren auf einer leicht reduzierten Stichprobe, basierend auf jenen Personen, die gültige Werte für die Skala „Langfristiger Erfolg“ $(N=477)$ aufweisen. In dieser bereinigten Stichprobe sind 41,9\% der befragten Personen $(N=200)$ weiblich, das Durchschnittsalter beträgt 38,4 Jahre (SD=9,5 Jahre).

\subsection{Erhebungsinstrument}

In dieser Phase des Projekts wurden die Konstrukte mit den ungekürzten Originalskalen erhoben. In Tab. 1 sind die Erhebungsinstrumente mit statistischen Kennwerten dokumentiert. Der kurz- und der langfristige Erfolg wurden gemessen mit dem 4QTE-Instrument von Grohmann und Kauffeld (2013). Damit kann der Forderung nach objektiver Messung von Transfererfolg nicht entsprochen werden, die stark an Inhalten orientiert sein müsste und eine genügend große Stichprobe an Teilnehmenden eines spezifischen Kurses erfordert, was in der Praxis selten anzutreffen ist. Das Instrument ermöglicht die subjektive Erfassung der Wirkung in einer generischen Art, so dass verschiedene Weiterbildungen gleichzeitig in die Analysen eingehen können.

Tab. 1 Konstrukte und statistische Kennwerte

\begin{tabular}{|c|c|c|c|c|c|}
\hline & $\begin{array}{l}\text { Anzahl } \\
\text { Items }\end{array}$ & $\begin{array}{l}\text { Anzahl Items } \\
\text { Beispielitem }\end{array}$ & Reliabilität & $N$ & $\mathrm{M}(\mathrm{SD})$ \\
\hline Kurzfristiger Erfolg $^{a}$ & 6 & $\begin{array}{l}\text { Im Kurs habe ich sehr viel } \\
\text { Neues gelernt }\end{array}$ & 0,878 & 469 & $\begin{array}{l}5,92 \\
(0,95)\end{array}$ \\
\hline Langfristiger Erfolg $^{\mathrm{a}}$ & 6 & $\begin{array}{l}\text { Es gelingt mir sehr gut, die } \\
\text { erlernten Inhalte in meiner } \\
\text { täglichen Arbeit anzuwenden }\end{array}$ & 0,915 & 477 & $\begin{array}{l}5,00 \\
(1,23)\end{array}$ \\
\hline $\begin{array}{l}\text { Lernfeld: methodi- } \\
\text { sche Gestaltung }{ }^{b}\end{array}$ & 6 & $\begin{array}{l}\text { Die im Kurs eingesetzten Me- } \\
\text { thoden unterstützten das Lernen }\end{array}$ & 0,883 & 476 & $\begin{array}{l}6,30 \\
(0,68)\end{array}$ \\
\hline $\begin{array}{l}\text { Lernfeld: Transfer- } \\
\text { orientierung }{ }^{b}\end{array}$ & 5 & $\begin{array}{l}\text { Was im Kurs besprochen wur- } \\
\text { de, ist Situationen meiner Ar- } \\
\text { beit ähnlich }\end{array}$ & 0,918 & 475 & $\begin{array}{l}5,87 \\
(0,98)\end{array}$ \\
\hline $\begin{array}{l}\text { Lernfeld: Kompetenz- } \\
\text { unterstützung }^{\text {b }}\end{array}$ & 5 & $\begin{array}{l}\text { Im Kurs wurde ich sachlich } \\
\text { über meine Fortschritte infor- } \\
\text { miert }\end{array}$ & 0,921 & 461 & $\begin{array}{l}5,64 \\
(1,11)\end{array}$ \\
\hline $\begin{array}{l}\text { Lernfeld: } \\
\text { Situiertheit }^{\mathrm{b}}\end{array}$ & 4 & $\begin{array}{l}\text { Im Kurs wurde an das Vor- } \\
\text { wissen der Teilnehmenden } \\
\text { angeknüpft }\end{array}$ & 0,886 & 477 & $\begin{array}{l}6,06 \\
(0,89)\end{array}$ \\
\hline $\begin{array}{l}\text { Funktionsfeld: Trans- } \\
\text { ferunterstützung } \\
\text { durch vorgesetzte } \\
\text { Person }{ }^{\text {b }}\end{array}$ & 7 & $\begin{array}{l}\text { Mein Vorgesetzter erkundigt } \\
\text { sich von Zeit zu Zeit, wie ich } \\
\text { das Gelernte in meiner Arbeit } \\
\text { umsetze }\end{array}$ & 0,966 & 468 & $\begin{array}{l}4,94 \\
(1,67)\end{array}$ \\
\hline $\begin{array}{l}\text { Funktionsfeld: Trans- } \\
\text { ferklima im Team }{ }^{b}\end{array}$ & 6 & $\begin{array}{l}\text { In unserem Team sind wir da- } \\
\text { ran interessiert, unsere Arbeits- } \\
\text { methoden ständig zu verbessern }\end{array}$ & 0,922 & 466 & $\begin{array}{l}5,27 \\
(1,35)\end{array}$ \\
\hline $\begin{array}{l}\text { Funktionsfeld: Trans- } \\
\text { ferkapazität der } \\
\text { Situation }^{\text {b }}\end{array}$ & 5 & $\begin{array}{l}\text { Meine Arbeitsbelastung er- } \\
\text { laubt es mir, das neu Gelernte } \\
\text { auszuprobieren }\end{array}$ & 0,850 & 475 & $\begin{array}{l}5,47 \\
(1,08)\end{array}$ \\
\hline
\end{tabular}

${ }^{\mathrm{a}}$ Grohmann und Kauffeld (2013)

${ }^{b}$ Hinrichs (2014); Kauffeld et al. (2008) 
Die unabhängigen Variablen des Lern- und Funktionsfelds wurden mit den Skalen von Hinrichs (2014) gemessen, die aufbauend auf den Vorarbeiten von Gessler (2012) eine Kürzung des GLTSI (Kauffeld et al. 2008) vorgenommen und die neuen Skalen validiert hat. Alle Items wurden mittels siebenstufigen Likert-Skalen abgefragt (von 1 „trifft gar nicht zu“ bis 7 ,trifft völlig zu“).

\subsection{Auswertungsmethode}

Die nachfolgende Analyse hatte das Ziel, die Hypothesen in der Gesamtstichprobe zu prüfen, also eine generelle Aussage darüber zu machen, ob es einen Zusammenhang zwischen den Faktoren des Lern- und des Funktionsfelds für den subjektiv wahrgenommenen kurz- und langfristigen Erfolg gibt, unabhängig von Firma, Weiterbildungsthema und weiteren Moderatoren.

In einem ersten Schritt wurde deskriptiv analysiert, wie der kurz- und langfristige Erfolg eingeschätzt wurden. Danach wurde mittels multiplen linearen Regressionen geprüft, wie groß der Zusammenhang ist zwischen den Faktoren des Lern- und Funktionsfelds mit der Einschätzung des kurz- und langfristigen Erfolgs. Mit der ENTER-Methode wurde zuerst ein Modell mit allen Faktoren des Lernfelds, dann ein Modell mit allen Faktoren des Funktionsfelds und zum Abschluss ein Gesamtmodell mit allen Faktoren berechnet. Durch diesen simultanen Einbezug war es möglich einzuschätzen, a) welche Faktoren zentral bleiben, wenn andere Faktoren in die Gleichung eingehen und b) konnte untersucht werden, wie gut die Faktoren geeignet sind, Varianz des kurz- bzw. langfristigen Erfolges einer Weiterbildung vorherzusagen (Baltes-Götz 2006). Alle statistischen Analysen wurden mit SPSS für Windows Version 24 durchgeführt.

\section{Ergebnisse}

Es zeigte sich, dass die Teilnehmenden den kurzfristigen Erfolg höher einschätzten als den langfristigen Erfolg ( $\mathrm{T}=21,8(468), p<0,001)$. Am positivsten fiel dabei die Subdimension der kurzfristigen Reaktion aus, d.h. die Zufriedenheit mit der Weiterbildung und die Einschätzung von deren Nützlichkeit. Die Einschätzung des langfristigen Erfolgs war deutlich niedriger, insbesondere die Dimension der Auswirkungen auf die Organisation (s. Abb. 2).

In der multiplen Regressionsanalyse werden die Zusammenhänge simultan geschätzt. Dabei wurden im ersten Schritt die relevanten Einflussfaktoren für den kurzfristigen Erfolg untersucht. In Modell $1^{1}$ wurden die Faktoren des Lernfelds analysiert. Alle wiesen einen signifikanten Zusammenhang auf, außer die Situiertheit der Weiterbildung (s. Tab. 2). Die methodische Gestaltung (beta $=0,335 ; p<0,001$ ) und die Transferorientierung (beta $=0,331 ; p<0,001$ ) hatten dabei vergleichbar hohe Effekte. Zusammen erklärten die drei signifikanten Faktoren 47,3\% der Streuung des kurzfristigen Erfolgs (Modell 1). Betrachtet man die Effekte der Faktoren des Funktionsfelds (Modell 2), wurden nur die Transferunterstützung durch die vorgesetzte

\footnotetext{
${ }^{1}$ Die Voraussetzungen von Regressionsanalysen wurden geprüft.
} 


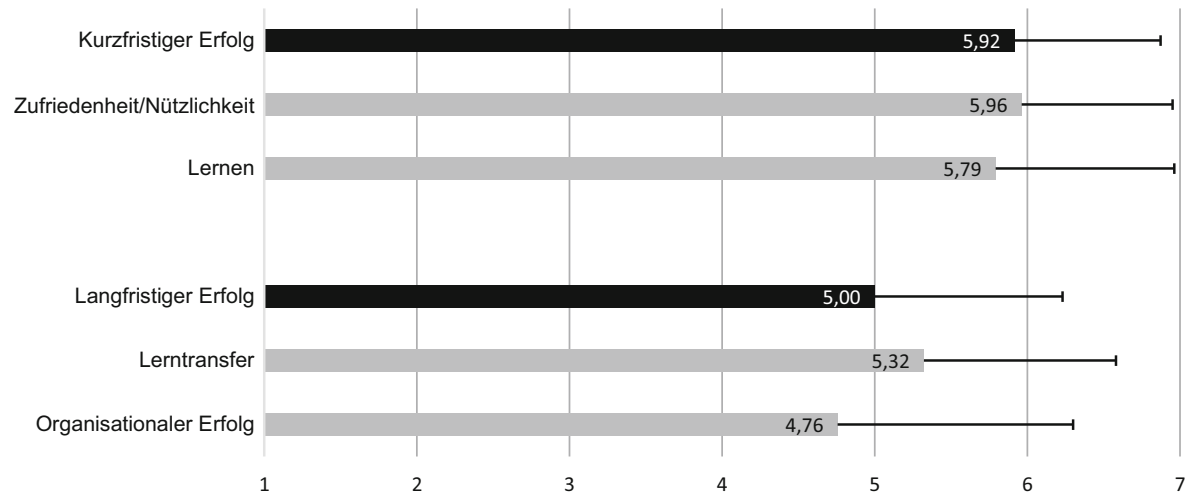

Abb. 2 Kurz- und langfristiger Erfolg mit Subdimensionen $(N=477)$

Tab. 2 Resultate der linearen Regression für kurzfristigen Erfolg

\begin{tabular}{llll}
\hline & \multicolumn{2}{l}{ AV: kurzfristiger Erfolg } & \\
& Modell 1 & Modell 2 & Modell 3 \\
\hline Konstante $(S R)$ & $0,35(0,31)$ & $3,62(0,21)$ & $-0,12(0,33)$ \\
LF: methodische Gestaltung & $0,335 * * *$ & - & $0,357 * * *$ \\
LF: Transferorientierung & $0,331 * * *$ & - & $0,227 * *$ \\
LF: Kompetenzunterstützung & $0,173 * * *$ & - & $0,094 *$ \\
LF: Situiertheit & $\mathrm{ns}$ & - & $\mathrm{ns}$ \\
FF: Transferunterstützung Chef & - & $0,159 * * *$ & $\mathrm{~ns}$ \\
FF: Transferklima Team & - & $\mathrm{ns}$ & $\mathrm{ns}$ \\
FF: Transferkapazität der Situation & - & $0,397 * * *$ & $0,193 * * *$ \\
R ${ }^{2}$ korrigiert & 0,473 & 0,243 & 0,500 \\
F(df1/df2) & $102,60(4 / 449)$ & $49,14(3 / 447)$ & $63,23(7 / 429)$ \\
\hline
\end{tabular}

two-tailed tests

$* p<0,05$

$* * p<0,01$

$* * * \quad p<0,001$

$n s$ nicht signifikant

Person (beta $=0,159 ; p<0,001$ ) und die Transferkapazität (beta $=0,397 ; p<0,001$ ) signifikant und erklärten gemeinsam $24,3 \%$ der Streuung. Betrachtet man alle Faktoren simultan (Modell 3), wird deutlich, dass der kurzfristige Erfolg am stärksten zusammenhing mit der methodischen Gestaltung (beta $=0,357 ; p<0,001$ ) und der Transferorientierung der Weiterbildung (beta $=0,227 ; p<0,001$ ). Die Transferkapazität der Situation im Funktionsfeld trug zur zusätzlichen Varianzaufklärung bei (beta $=0,193 ; p<0,001$ ), die übrigen Faktoren des Funktionsfelds waren nicht signifikant.

Hypothese 1 kann damit bestätigt werden. Das Lernfeld stellte sich in dieser Studie als relevant für die subjektive Einschätzung des kurzfristigen Erfolgs heraus. War das Lernfeld gut gestaltet, so erhöhte dies die Zufriedenheit der Teilnehmenden, deren Einschätzung, dass die Teilnahme am Training nützlich war, und das Gefühl, etwas Neues gelernt zu haben. Hypothese 3, dass das Funktionsfeld wenig 
Tab. 3 Resultate der linearen Regression für langfristigen Erfolg

\begin{tabular}{llll}
\hline & \multicolumn{2}{l}{ AV: langfristiger Erfolg } & \\
& Modell 1 & Modell 2 & Modell 3 \\
\hline Konstante $(S R)$ & $0,45(0,49)$ & $0,31(0,24)$ & $-1,31(0,43)$ \\
LF: methodische Gestaltung & $\mathrm{ns}$ & - & $\mathrm{ns}$ \\
LF: Transferorientierung & $0,317 * * *$ & - & $0,136^{* *}$ \\
LF: Kompetenzunterstuitzung & $0,312^{* * *}$ & - & $0,120^{* *}$ \\
LF: Situiertheit & $\mathrm{ns}$ & - & $\mathrm{ns}$ \\
FF: Transferunterstuitzung Chef & - & $0,279 * * *$ & $0,213 * * *$ \\
FF: Transferklima Team & - & $0,157 * * *$ & $0,163^{* * *}$ \\
FF: Transferkapazität der Situation & - & $0,412^{* * *}$ & $0,320 * * *$ \\
R ${ }^{2}$ korrigiert & 0,309 & 0,491 & 0,549 \\
F(df1/df2)= & 52,36 & $147,99(3 / 454)$ & $90,66(6 / 436)$ \\
& $(4 / 455) * * *$ & & \\
\hline
\end{tabular}

two-tailed tests

$* p<0,05$

$* * p<0,01$

$* * * p<0,001$

ns nicht signifikant

Einfluss hat auf den kurzfristigen Erfolg, kann auf der Grundlage der Daten dieser Studie nicht angenommen werden, da die Transferkapazität der Situation zusätzliche Varianz des kurzfristigen Erfolgs erklärte.

Wenn man den langfristigen Erfolg betrachtet (s. Tab. 3), zeigt sich in Modell 1, dass die methodische Gestaltung und die Situiertheit der Weiterbildung keine signifikanten Effekte aufweisen, die Transferorientierung (beta $=0,317 ; p<0,001$ ) und die Kompetenzunterstützung (beta $=0,312 ; p<0,001$ ) dagegen schon. Die beiden Faktoren erklärten gemeinsam 30,9\% der Varianz des langfristigen Erfolgs. In Modell 2 ist ersichtlich, dass alle drei Faktoren des Funktionsfelds in einem signifikanten Zusammenhang mit dem langfristigen Erfolg standen. Diese blieben auch signifikant in Modell 3, der simultanen Testung aller Faktoren, wobei die Transferkapazität der Situation (beta $=0,320 ; p<0,001$ ) der wichtigste Faktor war, gefolgt von der Transferunterstützung durch den Vorgesetzten (beta $=0,213 ; p<0,001$ ) und dem Transferklima im Team (beta $=0,163 ; p<0,001$ ). Die Transferunterstützung und die Kompetenzunterstützung im Lernfeld trugen ebenfalls zur Varianzaufklärung im Gesamtmodell bei.

Damit kann Hypothese 2 angenommen werden. Die Transferorientierung und die Kompetenzunterstützung in der Weiterbildung erklären den langfristigen Erfolg einer Weiterbildung. Für diesen Erfolg aber zusätzlich grundlegend sind die Faktoren des Funktionsfelds; demzufolge konnte Hypothese 4 ebenfalls bestätigt werden.

\section{Diskussion, praktische Implikationen und Ausblick}

Durch die vorliegende Studie sollte der Frage nachgegangen werden, was den Erfolg einer Weiterbildung ausmacht und von welchen gestaltbaren Faktoren dieser Erfolg abhängt. Der Forderung von Grossman und Salas (2011) folgend, ging es 
in dieser Studie nicht darum, möglichst umfassend zu analysieren, was den Erfolg einer Weiterbildung maximiert, sondern danach zu fragen, was die wichtigsten, von Organisationen gestaltbaren Faktoren sind, die den Erfolg einer Weiterbildung positiv beeinflussen können. Diese Faktoren wurden entlang von Gesslers (2012) Rahmenmodell gruppiert, d.h. es wurde unterschieden zwischen zentralen Merkmalen des Lernfelds (Trainings- und Lernbedingungen) und grundlegenden Attributen des Arbeitsumfelds, in dem das Gelernte angewendet werden soll.

Die zweite Differenzierung erfolgte, indem der Erfolg basierend auf den theoretischen Überlegungen von Grohmann und Kauffeld (2013) als kurzfristiger Erfolg (Zufriedenheit, Nützlichkeitseinschätzung und Lernen) und langfristiger Erfolg (Lerntransfer in den beruflichen Kontext und Auswirkungen auf die Organisation) gemessen wurden.

Aufgrund bisheriger theoretischer und empirischer Befunde war zu erwarten, dass die Trainings- und Lernbedingungen (Lernfeld) die Grundlage für den kurzfristigen Erfolg legen. Es wurde angenommen, dass die methodische Gestaltung und die Situiertheit der Weiterbildung, die Kompetenzunterstützung und die Transferorientierung in der Weiterbildung, wie zufrieden die Teilnehmenden mit der Weiterbildung sind, ob sie die Teilnahme als nützlich einschätzen und das Gefühl haben, etwas gelernt zu haben (Hypothese 1). Dies bestätigten die Analysen weitgehend, denn die Lernfeldfaktoren erklärten gemeinsam 47,3\% der Varianz des kurzfristigen Erfolgs. Dabei wurde die Situiertheit der Weiterbildung nicht signifikant. Die vier Faktoren lassen sich zwar messtheoretisch voneinander unterscheiden, korrelieren jedoch hoch miteinander und bei einer simultanen Überprüfung ihres Zusammenhangs mit dem kurzfristigen Erfolg führte dies dazu, dass die Situiertheit der Weiterbildung keine zusätzliche Varianz aufklären konnte.

Aufgrund der dargestellten bisherigen Befunde wurde in dieser Studie zudem die Hypothese aufgestellt, dass die Faktoren Kompetenzorientierung und die Transferorientierung der Weiterbildung auch Effekte auf den langfristigen Erfolg einer Weiterbildung haben. Werden im Training individualisiert Kompetenzen aufgebaut (Kompetenzorientierung) und die Inhalte auf das zukünftige Praxisfeld ausgerichtet (Transferorientierung), so werden die Anwendung unterstützt und damit auch mögliche organisationale Resultate ermöglicht (Hypothese 2).

Dies konnte durch die vorliegenden Daten bestätigt werden, was die Befunde aus zahlreichen Studien repliziert und bestätigt (Kauffeld et al. 2008; Grossman und Salas 2011; Burke und Hutchins 2008).

Neben der guten Gestaltung der Weiterbildung wurde des Weiteren angenommen, dass der langfristige Erfolg einer Weiterbildung in Zusammenhang damit steht, inwieweit das Gelernte im beruflichen Umfeld angewendet werden kann (Transferkapazität der Situation) und inwieweit der Vorgesetzte und das Team diese Anwendung ermöglichen und unterstützen (Hypothese 4). Diese Hypothese konnte durch die vorliegende Studie bestätigt werden. Die drei genannten Faktoren erklärten knapp 50\% der Varianz des langfristigen Erfolgs. Damit werden die Befunde von Gessler (2012) und Hinrichs (2016) und weiteren Studien (Blume et al. 2010) bestätigt.

Für den kurzfristigen Erfolg stellten sich diese Faktoren erwartungsgemäß als weniger bedeutsam heraus (Hypothese 3). Dass in den Analysen für die Transferkapazität der Situation ein signifikanter Zusammenhang mit dem langfristigen Erfolg 
festgestellt werden konnte, kann darauf zurückgeführt werden, dass die Teilnehmenden sowohl den kurz- als auch den langfristigen Erfolg zum gleichen Zeitpunkt beurteilen mussten. Die Ergebnisse könnten anders aussehen, wenn der kurzfristige Erfolg wirklich unmittelbar nach der jeweiligen Weiterbildung gemessen werden würde. Dies bleibt in zukünftigen Studien zu prüfen.

Die vorliegende Studie liefert basierend auf querschnittlich erhobenen Daten aus verschiedenen betrieblichen Weiterbildungsmaßnahmen Evidenzen dafür, dass der kurz- und der langfristige Erfolg von Weiterbildungen von unterschiedlichen Faktoren abhängen. Die Gestaltung der Weiterbildung ist insbesondere relevant für die Zufriedenheit und den gefühlten Lernerfolg. Will man hingegen den langfristigen Erfolg einer Weiterbildung positiv beeinflussen, ist die Gestaltung der Weiterbildung zwar auch wichtig, noch relevanter ist jedoch die Gestaltung des beruflichen Umfelds. Der Lerntransfer und organisationale Auswirkungen sind abhängig von der Möglichkeit, das Gelernte anzuwenden, vom Support durch die vorgesetzten Personen und dem Transferklima im Team.

Dieser Befund hat direkte praktische Implikationen für Organisationen, da diese Faktoren durch die Unternehmen gestaltbar sind. Werden sie bei der Planung und Konzeption berücksichtigt, kann damit der Wert von betrieblichen Weiterbildungen erhöht werden. Dies adressiert eine zentrale aktuelle Herausforderung in der Praxis (Fandel-Meyer et al. 2015). Konkret scheint es angesichts der Ergebnisse angezeigt, dass die zentrale Funktion von Führungskräften für den Lerntransfer und damit den Erfolg von betrieblichen Weiterbildungen in Führungsseminaren gezielt thematisiert wird.

Bei der Diskussion der vorliegenden Ergebnisse ist zu berücksichtigen, dass sie auf querschnittlichen Daten basieren, was bedeutet, dass lediglich von Zusammenhängen und nicht von Wirkungen ausgegangen werden kann. Dadurch, dass alle erhobenen Merkmale auf subjektiven Einschätzungen der Teilnehmenden basieren, werden die festgestellten Effekte vermutlich überschätzt (Blume et al. 2010). Die von Gessler (2012) postulierte Interaktion von Lern- und Funktionsfeld konnte mit den vorliegenden Daten nicht modelliert werden. Zum Beispiel konnte nicht gezeigt werden, inwiefern die Transferorientierung eines Trainings die Fähigkeit eines Individuums erhöht, Transfersituationen im Funktionsfeld zu erkennen und zu gestalten. Der Einfluss, den Merkmale der Trainingsteilnehmenden auf den kurz- und langfristigen Erfolg haben, wurde in den vorliegenden Analysen bewusst nicht untersucht, da der Fokus auf unmittelbar durch die Organisation gestaltbaren Faktoren lag. Welche Effekte einzelne Merkmale der Teilnehmenden auf den Erfolg von Weiterbildung haben, muss hier offen bleiben. Gerade die Frage nach der Bedeutsamkeit der Transfermotivation der Teilnehmenden ist jedoch in zukünftigen Studien zu prüfen, da die Transfermotivation in anderen Studien als Mediator für Weiterbildungserfolg identifiziert wurde (Hinrichs 2016; Seyler et al. 1998).

Auf der Grundlage dieser Studie kann festgehalten werden, dass der Erfolg und damit der Wert von Weiterbildungen von verschiedenen durch die Unternehmen gestaltbaren Faktoren abhängen. Es ist deshalb für Organisationen lohnenswert, neben der Zufriedenheit auch die subjektive Einschätzung des langfristigen Erfolgs und die Bewertung von spezifischen Faktoren im Lern- und im Funktionsfeld zu evaluieren. Mit solchen Evaluationen schaffen sie die Grundlage dafür, differenziert zu 
analysieren, an welchen Stellschrauben gedreht werden muss, um den Erfolg und den Wert ihrer Weiterbildungen zu verbessern.

Open Access Dieser Artikel wird unter der Creative Commons Namensnennung 4.0 International Lizenz (http://creativecommons.org/licenses/by/4.0/deed.de) veröffentlicht, welche die Nutzung, Vervielfältigung, Bearbeitung, Verbreitung und Wiedergabe in jeglichem Medium und Format erlaubt, sofern Sie den/die ursprünglichen Autor(en) und die Quelle ordnungsgemäß nennen, einen Link zur Creative Commons Lizenz beifügen und angeben, ob Änderungen vorgenommen wurden.

\section{Literatur}

Baldwin, T.T., \& Ford, J.K. (1988). Transfer of training: a review and directions for future research. Personnel Psychology, 41(1), 63-105.

Baltes-Götz, B. (2006). Lineare Regressionsanalyse mit SPSS. Universitäts-Rechenzentrum Trier. http:// www.uni-trier.de/urt/user/baltes/docs/linreg/linreg.pdf. Zugegriffen: 27. Apr. 2018.

Bhatti, M. A., Ali, S., Isa, M.F.M., \& Battour, M.M. (2014). Training transfer and transfer motivation: the influence of individual, environmental, situational, training design, and affective reaction factors. Performance Improvement Quarterly, 27(1), 51-82.

Blume, B. D., Ford, J. K., Baldwin, T. T., \& Huang, J. L. (2010). Transfer of training: a meta-analytic review. Journal of Management, 39, 1065-1105.

Bundesamt für Statistik (2014). Berufliche Weiterbildung in Unternehmen der Schweiz - Ergebnisse der Erhebung zur beruflichen Weiterbildung in Unternehmen (SBW) 2011. Neuchâtel: Eidgenössisches Departement des Innern EDI.

Burke, L. A., \& Hutchins, H. M. (2008). A study of best practices in training transfer and proposed model of transfer. Human Resource Development Quarterly, 19, 107-128.

Chatterjee, A., Pereira, A., \& Bates, R. (2018). Impact of individual perception of organizational culture on the learning transfer environment. International Journal of Training and Development, 22(1), 15-33.

Clarke, N. (2002). Job/work environment factors influencing training effectiveness within a human service agency: some indicative support for Baldwin and Ford's transfer climate construct. International Journal of Training and Development, 6, 146-162.

Colquitt, J. A., LePine, J.A., \& Noe, R.A. (2000). Toward an integrative theory of training motivation: a meta-analytic path analysis of 20 years of research. Journal of Applied Psychology, 85, 678-707.

Deci, E.L., \& Ryan, R. M. (1993). Die Selbstbestimmungstheorie der Motivation und ihre Bedeutung für die Pädagogik. Zeitschrift für Pädagogik, 39, 223-238.

Devos, C., Dumay, X., Bonami, M., Bates, R., \& Holton III, E. (2007). The Learning Transfer System Inventory (LTSI) translated into French: internal structure and predictive validity. International Journal of Training and Development, 11(3), 181-199.

Fandel-Meyer, T., Schneider, C., Seufert, S., Meier, C., \& Schuchmann, D. (2015). scil Trendstudie 2015/2016. Trends im Corporate Learning. St. Gallen: Swiss Centre for Innovations in Learning.

Festner, D. (2012). Veränderbarkeit betrieblichen Handelns. Transfer unter Einfluss von Merkmalen der Lernperson, der Lern- und Arbeitsumgebung. Frankfurt a.M.: Peter Lang.

Foxon, M. (1997). The influence of motivation to transfer, action planning, and manager support on transfer process. Performance Improvement Quarterly, 10(2), 42-63.

Gagné, R.M., Wager, W.W., Golas, K.C., \& Keller, J.M. (2005). Principles of instructional design (5. Aufl.). Belmont: Wadsworth/Thomson Learning.

Gessler, M. (2012). Lerntransfer in der beruflichen Weiterbildung - empirische Prüfung eines integrierten Rahmenmodells mittels Strukturgleichungsmodellierung. Zeitschrift für Berufs- und Wirtschaftspädagogik, 108(3), 364-393.

Grohmann, A., \& Kauffeld, S. (2013). Evaluating training programs: development and correlates of the Questionnaire for Professional Training Evaluation. International Journal of Training and Development, 17(2), 135-155.

Grossman, R., \& Salas, E. (2011). The transfer of training: what really matters. International Journal of Training and Development, 15(2), 103-120.

Hanke, U. (2005). Evaluation von Weiterbildung. Theoretische Überlegungen und praktische Ideen. Hamburg: Dr. Kovac.

Hattie, J. (2013). Visible learning: a synthesis of over 800 meta-analyses relating to achievement. London: Routledge. 
Hinrichs, A.-C. (2014). Predictors of collateral learning transfer in continuing vocational training. International Journal for Research in Vocational Education and Training, 1(1), 35-56.

Hinrichs, A.-C. (2016). Erfolgsfaktoren beruflicher Weiterbildung. Eine Längsschnittstudie zum Lerntransfer. Wiesbaden: Springer.

Holton III, E. F. (1996). The flawed four-level evaluation model. Human Resource Development Quarterly, $7,5-21$.

Holton III, E. F. (2005). Holton III's evaluation model: new evidence and construct elaborations. Advances in Developing Human Resources, 7, 37-54.

Holton III, E.F., Bates, R. A., \& Ruona, W.E. A. (2000). Development of a generalized learning transfer system inventory. Human Resource Development Quarterly, 11, 333-360.

Hutchins, H. M., Nimon, K., Bates, R., \& Holton III, E. (2013). Can the LTSI predict transfer performance? Testing intent to transfer as a proximal transfer of training outcome. International Journal of Selection and Assessment, 21(3), 251-263.

Käpplinger, B. (2009). Bildungscontrolling: Vor allem in Großbetrieben ein Thema - BIBB-Umfragen von 1997 und 2008 im Vergleich. In Bundesinstitut für Berufsbildung (Hrsg.), BIBB-Report 13/09. Bonn: Bundesinstitut für Berufsbildung (BIBB).

Kauffeld, S., Bates, R., Holton III, E. F., \& Müller, A. C. (2008). Das deutsche Lerntransfer-System-Inven$\operatorname{tar}$ (GLTSI): psychometrische Überprüfung der deutschsprachigen Version. Zeitschrift für Personalpsychologie, 7, 50-69.

Kirkpatrick, D. L. (1979). Techniques for evaluating training programs. Training and Development Journal, June, 178-192.

Lee, C., Lee, H., Lee, J., \& Park, J. (2014). A multiple group analysis of the training transfer model: exploring the differences between high and low performers in a Korean insurance company. The International Journal of Human Resource Management, 25, 2837-2857.

Lieberman, S., \& Hoffmann, S. (2008). The impact of practical relevance on training transfer. Evidence from a service quality training program for German bank clerks. International Journal of Training and Development, 12(2), 74-86.

Lim, D. H., \& Johnson, S. D. (2002). Trainee perceptions of factors that influence learning transfer. International Journal of Training and Development, 6, 36-48.

Lipowsky, F. (2004). Was macht Fortbildungen für Lehrkräfte erfolgreich? Die Deutsche Schule, 96, $462-479$.

Martin, H.J. (2010). Workplace climate and peer supports as determinants of training transfer. Human Resource Development Quarterly, 21, 87-104.

Massenberg, A.C., Schulte, E. M., \& Kauffeld, S. (2017). Never too early: learning transfer system factors affecting motivation to transfer before and after training programs. Human Resource Development Quarterly, 28(1), 55-85.

$\mathrm{Ng}, \mathrm{K} . \mathrm{H} .$, \& Ahm, R. (2011). The impact of the supervisors role in training programmes on the transfer of training: a case study in four east Malaysian local governments. Research and Practice in Human Resource Management, 19, 24-42.

Prenzel, M., \& Drechsel, B. (1996). Ein Jahr kaufmännische Erstausbildung: Veränderungen in Lernmotivation und Interesse. Unterrichtswissenschaft, 24(3), 217-234.

Scriven, M. (1991). Evaluation thesaurus. New York: SAGE.

Seyler, D. L., Holton III, E. F., Bates, R. A., Burnett, M.F., \& Carvalho, M.A. (1998). Factors affecting motivation to transfer training. International Journal of Training and Development, 2, 1-16.

Wang, G.G., \& Wilcox, D. (2006). Training evaluation: knowing more than is practiced. Advances in Developing Human Resources, 8(4), 528-539. 